as well. The first South Asian Federation of Endocrine Societies (SAFES) conference, held at Hyderabad, India, on 17 and 18 August 2013, acknowledged this reality.

Many of the topics discussed at the 2 days long conference, conducted in three parallel halls, covered the psychosocial and public health dimension of endocrinology, including diabetology.

These included the Presidential Oration, delivered by Dr. Sarita Bajaj, which called for national screening programs for congenital hypothyroidism, to tackle this easily preventable public endocrine health problem.

A session on gender and endocrinology focused upon the psychoneuroendocrine aspects of female sexuality, gender dysphoria and menopause. Psychosocial issues were also discussed at length in symposia including pediatric endocrinology and andrology.

In the sessions on diabetes, patient-oriented themes such as medical nutrition therapy, hypoglycemia and management during Ramadan were covered. The applicability of patientfriendly guidelines on the use of premixed insulin to South Asia was critically analyzed and a suggestion for regional guidelines made.

Other talks highlighted the public health impact of bone disorders and diabetes in South Asia, calling for action to prevent these endocrine morbidities.

Detailed emphasis on these issues is important, especially in South Asia, which bears the brunt of the pandemic of diabetes and its associated complications. SAFES' quest to be known as a global leader in endocrine care will not be achieved unless adequate attention is paid to the psychosocial dimension of health (without, of course, neglecting the biological domain).

The Scientific Committee, chaired by Dr. Subhankar Chowdhary (India), co-chaired by Dr. Faruque Pathan (Bangladesh) and ably assisted by Dr. Kaushik Pandit (India) and Dr. Sujoy Ghosh (India) deserves the credits for highlighting these important, yet often neglected, aspects of endocrine health.

\title{
Social health in endocrinology: A conference report
}

\author{
Department of Medicine, M.L.N. Medical College, Allahabad, \\ Uttar Pradesh, ${ }^{1}$ Osmania Medical College, \\ Hyderabad, Andhra Pradesh, India \\ Corresponding Author: \\ Dr. Sarita Bajaj,
}

Sir,

Psychosocial and public health related aspects of health are important not only in diabetes, but also in endocrinology 
Letters to Editor

\begin{tabular}{|l|l|}
\hline \multicolumn{2}{|c|}{ Access this article online } \\
\hline Quick Response Code: & Website: \\
\hline & www.joshd.net \\
\hline & \\
\hline
\end{tabular}

